\title{
Repeated yield drop phenomena as a cooperative effect
}

\author{
G ANANTHAKRISHNA \\ Materials Science Laboratory, Reactor Research Centre, Kalpakkam 603 102, India
}

\begin{abstract}
We present a theoretical model of repeated yielding (RY) which reproduces many experimentally observed features, apart from showing how the temporal behaviour of the phenomenon emerges as a consequence of the cooperative behaviour of defects. We first consider the case of step-like creep curves. Our model leads to a coupled set of nonlinear differential equations which admit limit cycle solutions, and thence jumps on the creep curve. Approximate closed form solutions for the limit cycles and the steps on the creep curve are cbtained. The model is then extended to the constant strain rate experiment by including the machine equation. The temporal ordering of $R Y$ is shown to follow, as well as several other features characteristic of RY. Chaotic fiow is also exhibited: the model has a sequence of perioddoubling bifurcations with an exponent equal to that of the quadratic map. Finally, we have analysed the fluctuations during the onset of RY using nonlinear Langevin equations. Fluctuations in the periodic ( $R$ : ) phase are also investigated. We conclude that $R Y$ is another example of a dissipative structure.
\end{abstract}

Keywords. Repeated yield drop; defects; cooperative behaviour; limit cycle solutions; nonlinear Langevin equations.

\section{Introduction}

There have been numerous phenomenological treatments of repeated yielding (RY) in the metallurgical literature (Bell 1973; Bodner and Rosen 1967; Cottrell 1953; McCormic 1972; Penning 1972; van den Beukel 1975, 1980). The best known model is Cottrell's dynamic strain-ageing model and its extensions (McCormic 1972; van den Beukel 1975, 1980). In these models, expressions are derived for such quantities as the critical strain, the critical strain rate, the dependence of the flow-stress on the strain rate, etc. Little attention has been paid to relating these quantities to the basic dislocation mechanisms such as cross glide, the Frank-Read mechanism, the formation of dislocation locks, etc. Also, there has been no attempt to investigate how the temporal behaviour of repeated yielding could arise naturally as a consequence of the basic dislocation mechanisms. From this point of view Cottrell's model is essentially static in character.

The transition from a single yield drop to a situation where RY occurs when certain 'drive' parameters (e.g., the strain rate, temperature, etc.), are varied has the physical features of a nonequilibrium phase transition. (For examples of such transitions which arise only when the system is driven away from equilibrium, see Nicolis and Prigogine 1977; Haken 1978). We have constructed dislocation-dynamical model (Ananthakrishna and Sahoo 1981; Ananthakrishna and Valsakumar 1982; Valsakumar and Ananthakrishna 1983) which exhibits most of the experimentally observed features of RY and demonstrates that RY is a nonequilibrium phase transition. Needless to say, we consider somewhat idealized conditions, and further do not attempt to fit any actual data. In $\S 2$, we start with the simplest case, namely, steps on the creep curve. Based on 
well-known dislocation mechanisms, we introduce a model which transforms one type of dislocation into another, giving rise to a set of coupled nonlinear differential equations for the corresponding densities. For a range of values of the rate constants, these equations are shown to admit limit cycle solutions. These lead immediately to jumps on creep curves. The model predicts a temperature range in which these jumps appear. In $\S 3$, we outline how approximate closed form solutions for the limit cycles and the steps on the creep curve are obtained. The results are shown to agree with experiments on zinc (Zagorukuyko et al 1977). Section 4 contains the extension of the model to the constant strain rate case by coupling the equations of $\$ 2$ to the machine equations. The temporal ordering of RY is shown to follow (Ananthakrishna and Valsakumar 1982). Also demonstrated are several important features typical of RY such as bounds on the strain rate, bounds on the concentration of solute atoms, the negative strain rate dependence of the flow stress, the dependence of the amplitude on the strain rate and strain, etc.

Our model also exhibits chaotic flow, which finds some support from experiments. We find a sequence of period doubling bifurcations (Ananthakrishna and Valsakumar $1983)$ with an exponent identical to that for a quadratic map. This is presented in $\S 5$. Section 6 is devoted to the study of fluctuations during the onset of RY using nonlinear Langevin equations. We have used the Monte-Carlo and Gaussian decoupling methods (Valsakumar et al 1983). As the strain rate approaches its critical value, the variance not only diverges, but also shows the periodic nature of the fluctuations. Fluctuations within the periodic phase (RY) are also investigated. Our entire analysis shows that RY is another example of a dissipative structure (Nicolis and Prigogine 1977). In the final section, some unresolved problems are discussed.

\section{Steps on the creep curve}

\subsection{The model}

Perhaps the simplest manifestation of instability in plastic flow, from a conceptual point of view, is a creep curve with steps. However, there are not many instances of measurements where stepped response has been observed (Ardley and Cottrell 1953; Navratil et al 1974; Stejskalova et al 1981; Zagorukuyko et al 1977; Da Silveira and Monteiro 1979; Lubahn and Felgar 1961). There appears to be no detailed theory for the phenomenon. The model we propose (Ananthakrishna and Sahoo 1981; Valsakumar and Ananthakrishna 1983) is a natural extension of our earlier work (Sahoo and Ananthakrishna 1982) where a theory of creep was developed under the assumption that mobile dislocations (denoted by $g$ ) and immobile dislocations (denoted by $s$ ) transform into each other and are in dynamical balance. In the present case, we introduce a third species of dislocations (denoted by $i$ ) which are surrounded by clouds of solute atoms. These are dislocations moving much slower than the mobile species, ultimately becoming immobile. The introduction of this species is reminiscent of the basic feature of Cottrell's (1953) theory, so that the model is in keeping with the essential spirit of Cottrell's theory.

Let $N_{g}, N_{s}$ and $N_{i}$ denote the densities of the $g, s$ and $i$ species respectively. The rate equations for these quantities are (a dot denotes the time derivative):

$$
\dot{N}_{g}=\theta V_{g} N_{g}-\mu N_{g}^{2}-\mu^{\prime} N_{g} N_{s}+\lambda N_{s}-\alpha N_{g},
$$




$$
\begin{aligned}
& \dot{N}_{s}=k \mu N_{g}^{2}-\mu^{\prime} N_{g} N_{s}-\lambda N_{s}+\alpha^{\prime} N_{i}, \\
& \dot{N}_{i}=\alpha N_{g}-\alpha^{\prime} N_{i} .
\end{aligned}
$$

Here $V$ denotes the dislocation velocity. We assume that $\theta, \bar{\mu}, \mu^{\prime}, \alpha, \alpha^{\prime}$, and $\lambda$ are constants for a given stress at a given temperature. The first term in (1) corresponds to the production of dislocation by the cross glide mechanism; the second corresponds to the immobilization of two mobile dislocations and the annihilation of pairs of dislocations at a rate $(l-k) \mu$; the third corresponds to the annihilation of a mobile dislocation with an immobile one; and the last arises from solute atoms gathering around dislocations. Once a certain number of solute atoms gather around a moving dislocation, its mobility is reduced, and it becomes a type $i$ dislocation (hence the source term $\alpha N_{g}$ in (3)). As the size of the solute atom cloud increases, the dislocation eventually becomes immobile (hence the source term $\alpha^{\prime} N_{i}$ in (2)). The term $\lambda N_{\mathrm{s}}$ comes from the (thermal or athermal) activation of immobile dislocations. The parameter $\alpha$ is expected to depend on the diffusion constant of the solute atoms, their concentration and the velocity of dislocations of type $i$. The parameter $\alpha^{\prime}$ is the rate of immobilization, and hence should be expected to depend on the critical velocity ( $k$ is a parameter close to unity).

It is convenient to make equations (1)-(3) dimensionless by setting

Then

$$
x=(\mu / \lambda) N_{\theta}, \quad y=\left(\mu / \theta V_{\theta}\right) N_{s}, \quad z=\left(\mu \alpha^{\prime} / \lambda \alpha\right) N_{i} .
$$

$$
\begin{aligned}
\mathrm{d} x / \mathrm{d} \tau & =(l-a) x-b x^{2}-x y+y, \\
\mathrm{~d} y / \mathrm{d} \tau & =b\left(k b x^{2}-x y-y+a z\right), \\
\mathrm{d} z / \mathrm{d} \tau & =c(x-z)
\end{aligned}
$$

where

$$
\lambda=\theta V_{g} t, \quad a=\alpha / \theta V_{g}, \quad b=\lambda / \theta V_{g}, \quad c=\alpha^{\prime} / \theta V_{g} .
$$

(We have set $\mu=\mu^{\prime}$ in order to reduce the number of parameters.)

\subsection{Stability analysis and existence of limit cycles}

Equations (5) (7) form a nonlinear system. Under well-known conditions (Minorsky 1962), these admit periodic solutions called limit cycles for a certain range of values of the parameters $a, b, c$ and $k$. Although the method of investigation is well known, for the sake of completeness we briefly outline the procedure used. Limit cycles are special classes of solutions which are isolated closed trajectories in the phase space $(x, y, z)$ such that any trajectory which is sufficiently close to it either approaches it or recedes from it. Such closed trajectories can arise only in nonlinear systems. The search for limit cycles is generally preceded and aided by an investigation of the properties of the system around the steady state values $x_{a}, y_{a}$, and $z_{a}$. The stability of the system is decided by the nature of its singular points--node, focus, saddle point or centre. Linearising the set of equations around $\left(x_{a}, y_{a}, z_{a}\right)$, one obtains an equation of the form

$$
\mathrm{d} \boldsymbol{\psi} / \mathrm{d} \tau=\boldsymbol{W} \boldsymbol{\psi}
$$

where $\psi$ is the column vector with components $\left(x-x_{a}\right),\left(y-y_{a}\right),\left(z-z_{a}\right)$, and $\boldsymbol{W}$ is a $3 \times 3$ matrix. A node arises when all three eigenvalues of $W$ are of the same sign, and a saddle point when only two of these are of the same sign; a focus occurs when there are two complex conjugate eigenvalues, and a centre when one eigenvalue is identically zero 
and the other two are pure imaginary. (In the last case the nature of the singular point may have to be analysed more carefully; see Minorsky 1962).

An attractive limit cycle exists if there is a surface surrounding an unstable focus into which all trajectories enter: in the present case, we first look for a domain in the parameter space $(a, b, c, k)$ when two of the eigenvalues are complex with at least one of them having a positive real part, and then show that such a surface exists (Ananthakrishna and Sahoo 1981). The only constraints that we have on the values of the parameters are $a<1$ (which arises because the total rate of production of dislocations is positive); $k<1$; and $a, b, c>0 .\left(N_{g}, N_{s}\right.$ and $N_{i}$ are obviously positive).

\subsection{Staircase creep}

An analytical solution of (5)-(7) for arbitrary values of the parameters, and valid for all times, is difficult. To obtain numerical solutions, $V_{g}$ must be known as a function of $N=N_{i}+N_{s}+N_{g}$ and $\sigma^{*}(N)$, where $\sigma^{*}$ is the effective stress. For simplicity we assume $V_{g}$ to be constant. Although this assumption is not physical, it can be argued that it will not alter the qualitative features of staircase creep (Ananthakrishna and Sahoo 1981). The creep curve obtained by integrating the Orowan equation is shown in figure 1 . The steps on the creep curve appear only in the secondary region, which is consistent with the existing experimental results (Zagorukuyko et al 1977; Da Silveira and Monteiro 1979).

Our model also predicts a feature which is in agreement with experiment, namely, that there are upper and lower bounds for the asymptotic creep rates for which staircase creep occurs. These arise because the frequency of oscillation $\sim V_{g} \operatorname{Im} \omega_{1}$, where $\omega_{1}$ is one of the two complex conjugate eigenvalues of $\boldsymbol{W}$. Bounds can be put on $\operatorname{Im} \omega_{1}$,



Figure 1. Staircase creep for typical values of the parameters $a, b$ and $c$. 
depending on the basic mechanism prevailing in the system. This implies corresponding bounds on $V_{g}$ in order to have observable steps on the creep curve. This in turn implies that the phenomenon can only be observed over a finite range of $\dot{\varepsilon}$ (in practice, somewhat less than three orders of magnitude). Moreover, since $V_{g}$ is temperature dependent (for a fixed stress), this phenomenon can only occur over a fixed range of temperature, as corroborated by the experimental results of Zagorukuyko et al (1977).

\section{Approximate solutions}

We outline an analytic procedure for obtaining asymptotic solutions to our equations. This is essential if one wants to relate the theoretical rate constants to accessible parameters such as the applied stress $\sigma_{a}$, temperature $T$, concentration of solute atoms $C$, etc. Our method follows that of Tyson (1977) for the BelousovZhabotinski oscillating chemical reaction. The main point is to identify a fast mode and adiabatically eliminate it to obtain a reduced set of two coupled equations from the original set of three equations (5)-(7). Approximate solutions are then obtained by using the method of relaxation oscillations (Minorsky 1962; Tyson 1977). Defining $X=x-X_{a}, Y=y-y_{a}, Z=z-z_{a}$, and $\tau^{\prime}=b \tau$, (5)-(7) may be re-written in the form

$$
\begin{aligned}
b \dot{X} & =-\left[\alpha X+\beta Y+b X^{2}+X Y\right], \\
\dot{Y} & =-\left[\gamma X+\delta Y-b X^{2}+X Y-a Z\right], \\
\dot{Z} & =\frac{c}{b}[X-Y],
\end{aligned}
$$

where the dot now refers to differentiation with respect to $\tau^{\prime}$. Further, the constants $\alpha, \ldots, \delta$ are

$$
\alpha=a+2 b x_{a}+y_{a}-1, \beta=x_{a}-1, \gamma=y_{a}-2 b x_{a}, \delta=x_{a}+1
$$

(The constant $\alpha$ used here is distinct from that appearing in (1) of $\S 2$.) Since $b \ll a, c$, we see that $|\dot{X}| \rightarrow \infty$ as $b \rightarrow 0$ unless the right side in (10) vanishes identically. This amounts to saying that $X$ must change with a characteristic time $\sim b$ to maintain the condition

$$
\alpha X+\beta Y+b X^{2}+X Y=0
$$

Thus $X$ is a fast variable that can be adiabatically eliminated from (10)-(12), and the resulting pair of independent equations used for further analysis.

We use the results of stability analysis and further express $\alpha, \beta, \gamma, \delta, x_{a}, y_{a}$ and $z_{a}$ as power series in $b$. We then determine the 'null clines'

$$
\dot{Y}=0 \leftrightarrow Z_{1}=Z(Y)=X(Y)+\frac{2}{a}\left[x_{a}+X(Y)\right] Y,
$$

and

$$
\dot{Z}=0 \leftrightarrow Z_{2}=X(Y),
$$

and again express $Z(Y)$ in powers of $b$. To find the limit cycles, we look for the intersection of the null clines in the region of negative slope. From the expressions for the null clines $Z_{1}$ and $Z_{2}$ we obtain a reasonable idea of the phase portrait. For $a<1 / 3$ and for $1 / \sqrt{2}<a<1$, the steady state is stable. For $1 / 3<a<1 / \sqrt{2}$, the null clines intersect in the negative slope region, which means that a limit cycle exists. Figure 2 


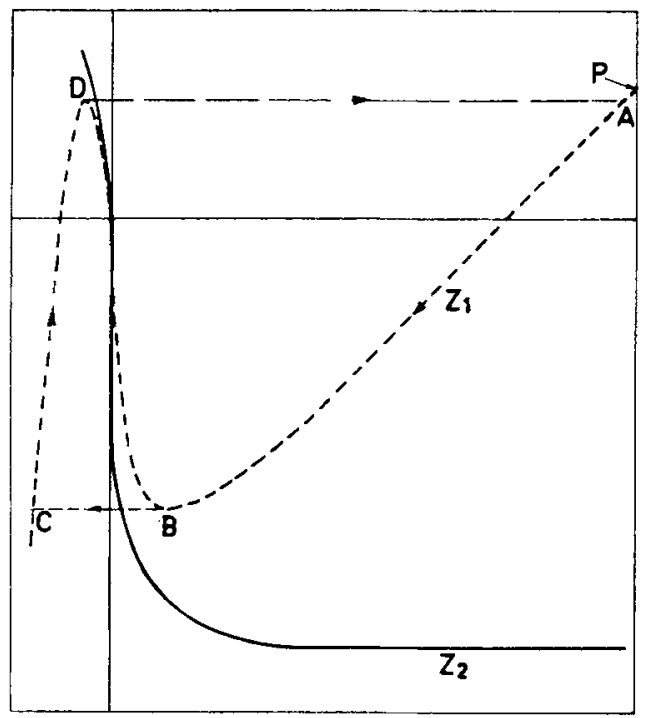

Figure 2. Phase plane portrait (the null clines $Z_{1}$ and $Z_{2}$ plotted as a function of $Y$ in the case $1 / 3<a$ $<1 / 2$. The limit cycle is ABCDA.

shows a typical phase portrait for $1 / 3<a<1 / 2$. Starting from an arbitrary point, the trajectory moves along the null cline $\dot{Y}=0$ until it reaches the turning point $D$, from where it almost instantaneously jumps to $A$. Thereafter it moves along the branch $\dot{Y}=0$ (slowly) until it reaches the second turning point $B$ from where it jumps to $C$, and the process continues. Thus the trajectory is a closed one and the limit cycle is $A \rightarrow B \rightarrow C$ $\rightarrow D \rightarrow A$. From the expressions for $Z_{1}$ and $Z_{2}$, it is possible to calculate also the period, the amplitude and the wave form of the limit cycle solution. Finally, as already stated, we can integrate the Orowan equation to obtain the steady state creep curve which exhibits steps (Valsakumar and Ananthakrishna 1983).

Although the results of the model (in its present form) are not directly applicable to the detailed results on zinc (Zagorukuyko et al 1977), they still permit qualitative comparison. That the steps are seen only in the secondary creep region is obviously consistent with our theory, since the latter predicts a bifurcation from the steady state solution to a periodic one. Zagorukuyko et al (1977) report a rapid monotonic increase of the magnitude of the step in the strain as a function of $\sigma_{a}$; a weak, decreasing dependence of the period of the jump on $\sigma_{a}$; and a decrease of the magnitude of the jump in strain with increasing $T$. All these features are consistent with our work.

\section{Repeated yield drop}

Our model can be extended to a constant strain rate experiment by augmenting equations (1)-(3) with the machine equation representing the load sensed by the load cell, namely

$$
\dot{\sigma}_{a}=K\left[\dot{\varepsilon}-b_{0}\left(N_{g}+\gamma N_{i}\right) V_{\theta}\left(\sigma^{*}\right)\right],
$$

where a dot denotes the time derivative. Here $\dot{\varepsilon}$ is the imposed strain rate, $K$ is the effective compliance, $b_{0}$ is the Burgers vector and $\sigma^{*}$ is the effective stress. The second term on the right in (17) is the plastic strain rate $\dot{\varepsilon}_{p}$. We assume the power law $V_{\theta}=V_{0}\left(\sigma^{*} / \sigma_{0}\right)^{m}$, with $\sigma^{*}=\sigma_{a}-H N^{1 / 2}$, where $H$ is a constant characteristic of 
hardening and $m$ is a velocity exponent. Following a procedure similar to that of $\S 2$, it can be shown that there is a domain in the space of the relevant parameters for which limit cycle solutions exist. Choosing values of the parameters in the domain of instability so as to be consistent with the expected values of the dislocation densities and the yield drops, numerical solutions of the system of equations may be obtained, and various characteristic features of the RY exhibited may be studied. These appear to be generally consistent with the experimental results. (Note that the latter are in the nature of averages over the sample dimensions.) We list these salient features: (a) There is a range of $\dot{\varepsilon}$ over which serrations are seen. (b) The model exhibits the negative strain rate behaviour of the flow stress at a fixed value of the plastic strain. The inset in figure 3 shows a typical plot of $\sigma_{a} v s \dot{\varepsilon}_{p}$, with a minimum in $\sigma_{a}$ at a point $\dot{\varepsilon}_{p}=\dot{\varepsilon}_{\min }$. Curves corresponding to larger $\varepsilon_{p}$ are displaced successively upwards. This feature has been both theoretically (Penning 1972; van den Beukel 1975) and experimentally verified (Bodner and Rosen 1967). (c) Figure 3 shows a typical plot of serrated yielding. The serrations are asymptotically periodic. Since it is not possible to identify them (from the plot itself) as serrations of type A or type B, a strain rate change test (Wijler and van Westrum 1971) must be carried out, from which it is found that beyond $\dot{\varepsilon}_{\min }$ the serrations are of type $B$. (d) The amplitude of the serration increases up to $\dot{\varepsilon}_{\min }$ and decreases thereafter. (e) The amplitude increases and saturates as a function of $\varepsilon$, consistent with experiments (McCormic 1971). (f) There are upper and lower bounds on the parameter $\alpha$ of (1) within which serrated yielding occurs. Since $\alpha$ depends on the concentration of solute atoms, this implies that there is a range of the solute atom concentration in which the phenomenon occurs. (g) $\varepsilon_{c}$ (the critical strain), as a function of $\dot{\varepsilon}$, first decreases and then increases (McCormic 1971). (h) Beyond the range of $\dot{\varepsilon}$ where serrated yielding occurs, the 'normal' behaviour of $\sigma_{a}(\varepsilon)$ is resumed.

It should be pointed out that there has so far been no attempt to derive the negative strain rate behaviour of flow stress (which is crucial for any meaningful description of the phenomenon) starting from dislocation inieractions. In the existing theories this is either assumed (Penning 1972) or derived (van den Beukel 1975) through a phenomenological treatment of waiting times involving in any case only individual dislocations. In contrast, this property emerges naturally in the present model from a consideration of dislocation interactions.

We have shown that the new temporal order represented by serrated yielding is the consequence of a bifurcation from a temporally homogeneous steady state plastic flow

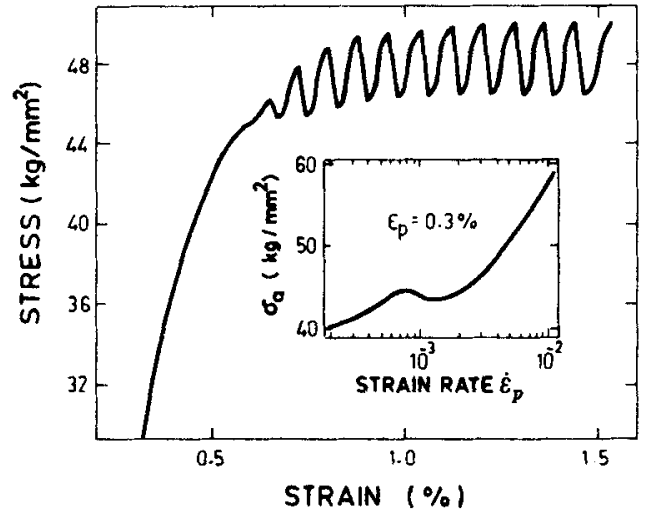

Figure 3. Calculated stress-strain curve showing repeated yielding. The inset is a graph $\sigma_{a}$ vs $\dot{\varepsilon}_{p}$ for the same values of the parameters. 
beyond some critical values of the parameters. This order is a result of a balance between the energy input (in the form of dislocation multiplication) and dissipation (annihilation, immobilisation and other processes). The phenomenon is obviously a far-from-equilibrium situation and is an example of a dissipative structure (Nicolis and Prigogine 1977).

\section{Chaotic flow}

We now turn to the chaotic flow exhibited by our model over a certain range of a drive parameter, the applied strain rate. (There is also some experimental evidence for such a flow, which will be discussed subsequently in brief). This adds to the growing list of models and physical situations exhibiting chaos (Ott 1981; Eckmann 1981; Lauterborn and Kramer 1981; Jefferies 1982). The model has an infinite sequence of perioddoubling bifurcation eventually leading to chaos. The region over which chaos is exhibited is very small compared to the range of $\dot{\varepsilon}$ over which RY is seen (RY is considered to be periodic). We have calculated the value of the associated exponent and found it to be the same as the exponent for the quadratic map. We have also obtained the associated one-dimensional map. As the parameter of interest is the applied strain rate $\dot{\varepsilon}$, we fix the values of all the other parameters within the instability region and study the bifurcation sequence with respect to the parameter $e=\dot{\varepsilon}\left(\mu / \lambda V_{0} b_{0}\right)$. The region where the period doubling bifurcation occurs is small, and is located near the upper end of the range of $e$ (the dimensionless strain rate) over which RY is observed. For the chosen values of the parameters, the first bifurcation from the periodic state with period $T$ to a state with a period $2 T$ occurs at $e_{1}=159.98444$, while the successive bifurcation to states with period $2^{2} T, 2^{3} T, \ldots$, occur at $e_{2}=173.7178, e_{3}=175.8974$, $\ldots$ The exponents $\delta=\lim \left(e_{n}-e_{n-1}\right) /\left(e_{n+1}-e_{n}\right)$ appears to be very close to that obtained by for the quadratic map (Grossman and Tnomae (1977), Feigenbaum (1978)). The estimated value of $e_{\infty}$ is 176.4669 , beyond which we find chaotic motion. Figures 4 and 5 show the variation of the stress $\sigma_{a}$ with time (equivalently, the strain) for $e=174.679$ (motion with period 4T) and $e=178.205$ (chaotic motion) respectively. A $\log -\log$ plot of the projection of the strange attractor in the $N_{g}-\sigma_{a}$ plane is shown in figure 6. The associated one-dimensional map is shown in figure 7. Unlike the corresponding map for the Lorenz model (Ott 1981), our map has a smooth rounded maximum similar to the quadratic map except that it is very much skewed.

The fact that our model can exhibit chaotic flow has prompted us to look for experimental evidence for the plots of repeated yield drops. (Of course, the value of the parameter which controls the magnitude of the variation in $\sigma_{a}$, i.e., the magnitude of the yield drop, has to be appropriately chosen). Even though we are constrained by the fact that the average stress level remains constant in our model, we have found evidence (Hall 1970) in support of such flows. Experimentally, too, this occurs towards the end of the $\dot{\varepsilon}$ range for which $\mathrm{RY}$ is seen. If we subtract the normally observed slow increase in the base level of the stress, there appear to be many more situations which perhaps correspond to chaos (Rosen and Bodner 1969).

\section{Fluctuations during the onset of repeated yielding}

We have discussed so far the possibility of a new temporal phase arising as a drive parameter (here, $\dot{\varepsilon}$ ) is varied. The situation corresponds to a hard mode instability in 

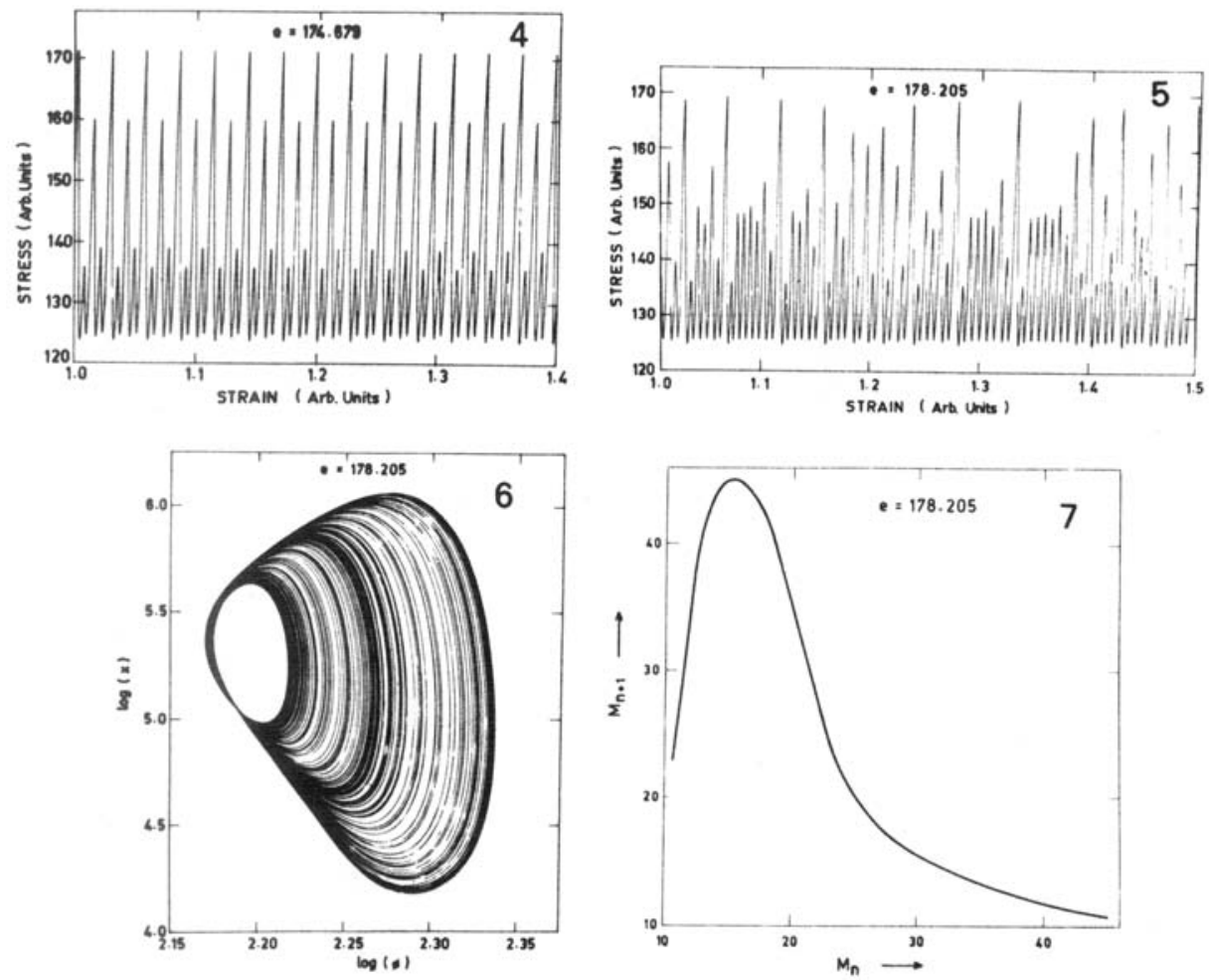

Figure 4. Stress-strain curve for $e=174.679$ with period 4T.

Figure 5. Stress-strain curve for $e=178 \cdot 205$, i.e., in the chaotic region.

Figure 6. A log-log plot of the projection of the strange attractor for $e=178.205$ in the $x-\phi$ plane. Here $x=(\mu / \lambda) N_{g}$ and $\phi=\sigma_{a} / \sigma_{0}$

Figure 7. The one-dimensional map associated with chaotic flow in the model.

which the real part of complex conjugate modes changes sign (from negative to positive) in the frequency plane, as the drive parameter crosses its critical value. The foregoing analysis is completely deterministic. We now analyse the nature of the fluctuations when the parameter is in the neighbourhood of its critical value.

Our analysis begins with the nonlinear Langevin equations obtained by adding Gaussian white noise terms to the deterministic equations. We have used both the Monte Carlo technique and a Gaussian decoupling method (Valsakumar et al 1983) to study the fluctuations. (However, in the present context the second method can be applied only in the special case $H=0$; otherwise powers of $N^{1 / 2}$ are present, which cannot be handled without further approximations). It is convenient to discuss the general features of the fluctuations with the help of the equivalent Fokker-Planck equation. The standard form of the Fokker-Planck equation is

$$
\frac{\partial \pi}{\partial t}(\xi, t)=\frac{\partial}{\partial \boldsymbol{\xi}}[(\boldsymbol{K}(t) \cdot \boldsymbol{\xi} \pi(\boldsymbol{\xi}, t))]+\frac{1}{2} \frac{\partial^{2}}{\partial \xi^{2}}[D(t) \pi(\xi, t)],
$$


where

$$
K(t)=\frac{\partial \mathbf{C}_{1}(\mathbf{y})}{\partial \mathbf{y}}, D(t)=C_{2}(\mathbf{y})
$$

and

$$
\frac{\mathrm{dy}}{\mathrm{d} t}=\mathrm{C}_{1}(y) \text { and } \xi=\varepsilon^{-1 / 2}[\mathbf{x}-\mathbf{y}]
$$

Here, $\mathbf{x}$ is the random variable, $\mathbf{y}$ its mean, $\mathbf{C}_{1}(\mathbf{y})$ the first jump moment and $\boldsymbol{C}_{2}(y)$ the second moment. ( $\boldsymbol{K}$ is the regression matrix.) $\varepsilon$ is the (system-size) expansion parameter. To identify the onset of the periodic state beyond the critical value of the drive parameter, it is convenient to use the concept of the irreversible circulation of fluctuation' defined by (Tomita and Tomita 1974; Tomita et al 1974)

$$
\boldsymbol{\alpha}=\frac{1}{2}\left[(\boldsymbol{K} \boldsymbol{\sigma})^{T}-\boldsymbol{K} \boldsymbol{\sigma}\right]
$$

where $\sigma$ is the variance ( $T$ represents the transpose). $K$ and $\sigma$ are related by

$$
\frac{1}{2} \sigma=\alpha+K \sigma+\frac{1}{2} D \text {. }
$$

If a periodic state occurs as a result of the hard mode instability both $\alpha$ and $\sigma$ diverge; the instability is incurred through $a$, which becomes increasingly large as the transition point is approached from below.

Our preliminary investigations reveal that as $\dot{\varepsilon} \rightarrow \dot{\varepsilon}_{c}$ from below, the fluctuations show an overall growth which is indicative of the divergence of the variance, in addition to exhibiting a near periodic modulation which is indicative of the approach to a periodic state. For $\dot{\varepsilon}>\dot{\varepsilon}_{c}$, the fluctuations have the normal characteristics expected when limit cycle solutions are supported. The details of this study will be reported elsewhere.

\section{Concluding remarks}

We have shown that the state of temporal order represented by steps on the creep curve or by repeated yield drops is a consequence of a bifurcation from a temporally homogeneous, steady-state plastic flow. (A similar approach has been used by Kubin et al (1984) for low temperature RY.) A major criticism that can be raised is that the theory does not take into account the inhomogeneous deformation that normally accompanies the phenomenon. (Although, to the best of the author's knowledge, experiments do not show which is the cause and which is the effect, i.e., whether an inhomogeneous deformation is the cause and a yield drop is the effect, or vice versa. We believe that it is not possible to disentangle the two. Nor is there any theory that takes the inhomogeneous deformation into proper account. The closest is that of Penning (1972) in which the feature is simply assumed). However, we argue (Ananthakrishna and Valsakumar 1982) that if the space dependence is properly taken into account, the inhomogeneity of the deformation should follow automatically. The point is that such an extended model would still exhibit the negative strain rate behaviour of the flow stress (which has been assumed by Penning (1972) to show that hopping and propagating band type solutions are supported). Attempts are underway to demonstrate explicitly that such solutions indeed occur when appropriate space dependence is included. 
Another possible criticism could be that the model merely 'mimics' the effects of the waiting of dislocations at obstacles which van den Beukel (1975) has used to derive $\sigma\left(\dot{\varepsilon}_{p}\right)$. However, this work deals with individual dislocations whereas what is observed in an actual experiment is an ensemble average over participating dislocations. Therefore, for such a theory to hold good, there must be a 'phase coherence' between the dislocations, if the effects are to manifest themselves at a gross level. Further, it is known that the negative strain rate behaviour is essential for RY. (For a detailed analysis of various tensile test conditions using the negative strain rate behaviour, see Neelakantan and Venkataraman 1983). Our analysis shows that this behaviour is a consequence of the competition between different dislocation interaction mechanisms. We have also checked (Ananthakrishna and Sahoo 1981) that the incorporation of certain other basic dislocation mechanisms (pile up, pinning and depinning of dislocations from obstacles) leads to similar results.

Finally, it should be pointed out that several materials like nylon (Schultz 1974), metallic glasses (Takayama 1979), wood, etc. are known to show RY. The present work suggests that the mathematical mechanism in all these cases must be the emergence of limit cycle solutions which are characteristic of non-linear systems. As plastic flow is basically nonlinear, such solutions are an intrinsic possibility. The basic microscopic physical mechanisms of course are bound to be different in different materials.

\section{Acknowledgement}

The author thanks Dr G Venkataraman for arousing his interest in this problem.

\section{References}

Ananthakrishna G and Sahoo D 1981 J. Phys. D14 2081

Ananthakrishna G and Valsakumar M C 1982 J. Phys. D15 L171

Ananthakrishna G and Valsakumar M C 1983 Phys. Lett. A95 69

Ardley G W and Cottrell A H 1953 Proc. R. Soc. A219 328

Bell J F 1973 Handbuch der Physik Band VIa/I (Berlin: Springer-Verlag)

Bodner S R and Rosen A 1967 J. Mech. Phys. Solids 1563

Cottrell A H 1953 Philos. Mag. 44829

Da Silveira T L and Monteiro S N 1979 Met. Trans. A10 1795

Eckmann J P 1981 Rev. Mod. Phys. 53643

Feigenbaum M J 1978 J. Stat. Phys. 1925

Grossman S and Tnomae S 1977 Z. Naturforsch. 32a 1353

Haken H 1978 Synergetics (Berlin: Springer-Verlag)

Hall E O 1970 Yield point phenomena in metals and alloys (Macmillan: London)

Jefferies D J 1982 Phys. Lett. A90 317

Kubin L P, Estrin Y and Spiesser Ph 1984 Res. Mechanica 1025

Lauterborn W and Kramer E 1981 Phys. Rev. Lett. 471445

Lubahn J D and Felgar R P 1961 Plasticity and creep of metals (New York: John Wiley)

McCormic P G 1971 Acta Metall. 19463

McCormic P G 1972 Acta Metall. 20351

Minorsky N 1962 Nonlinear oscillations (New Jersey: Van Nostrand)

Navratil V, Hammersky M and Blazkova 1974 Czech. J. Phys. B24 907

Neelakantan K and Venkataraman G 1983 Acta Met. 3177

Nicolis G and Prigogine I 1977 Self organization in non-equilibrium systems (New York: Wiley)

Ott E 1981 Rev. Mod. Phys. 53643

Penning P 1972 Acta Metall. 201169 
Rosen A and Bodner S K 1969 Mater. Sci. Eng. 4115

Sahoo D and Ananthakrishna G 1982 J. Phys. D15 1439

Schultz J 1974 Polymer materials science (New Jersey: Princeton-Hall)

Stejskalova V, Hammersky M, Luckac P, Vostry P and Sprusil B 1981 Czech J. Phys. B31 195

Takayama 1979 Scr. Met. 13463

Tomita K, Ohta T and Ohta H 1974 Prog. Theor. Phys. 521744

Tomita K and Tomita H 1974 Prog. Theor. Phys. 511731

Tyson J J 1977 J. Chem. Phys. 66905

Valsakumar M C and Ananthakrishna G 1983 J. Phys. D16 1055

Valsakumar M C, Murthy K P N and Ananthakrishna G 1983 J. Stat. Phys. 30637

van den Beukel A 1975 Phys. Status Solidi A30 197

van den Beukel A 1980 Acta Metall. 20965

Wijler A and van Westrum S 1971 Scr. Metall. 5531

Zagarukuyko L N, Ostasky A L and Soldatov V P 1977 Phys. Met. Metallogr. 43156

\section{Discussion}

P Rodriguez: You cited some results on zinc. In your model, the Cottrell type of solute locking is envisaged. What was the solute species?

G Ananthakrishna: Zinc is a poor example (the dislocations are actually produced due to inhomogeneities at the surface).

Rodriguez: Many of the conclusions with respect to the critical strain, the magnitude of the strain burst, the delay time for the strain burst to occur, etc., follow automatically once Cottrell locking is introduced. It is therefore not surprising that the model gets these right.

Ananthakrishna: Cottrell locking is not manifest in the equations of the model.

N Kumar: You get period doubling bifurcations even though your maps are not discrete. Is this because of the higher dimensionality of the system of equations?

Ananthakrishna: One-D are the simplest of systems which exhibit chaos. Coupled system of differential equations also exhibit such a behaviour. The associated 1-D map can be obtained.

C K Majumdar: It would be interesting to examine the structure of your set of equations from the point of view of catastrophe theory. 